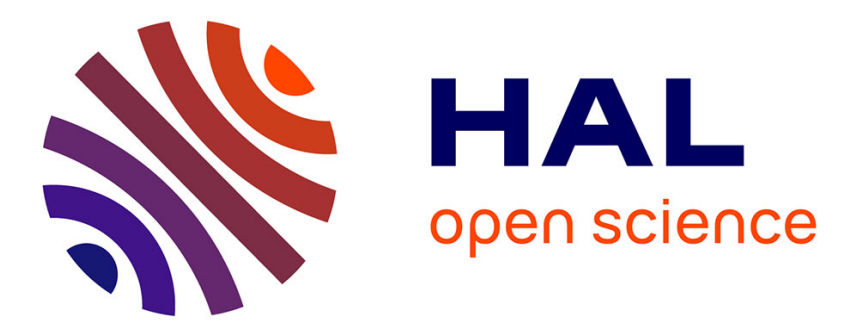

\title{
The Harmonic Balance Method with arc-length continuation in rotor/stator contact problems
}

Götz von Groll, David Ewins

\section{To cite this version:}

Götz von Groll, David Ewins. The Harmonic Balance Method with arc-length continuation in rotor/stator contact problems. Journal of Sound and Vibration, 2001, 10.1006/jsvi.2000.3298 . hal01333704

\section{HAL Id: hal-01333704 \\ https://hal.science/hal-01333704}

Submitted on 18 Jun 2016

HAL is a multi-disciplinary open access archive for the deposit and dissemination of scientific research documents, whether they are published or not. The documents may come from teaching and research institutions in France or abroad, or from public or private research centers.
L'archive ouverte pluridisciplinaire HAL, est destinée au dépôt et à la diffusion de documents scientifiques de niveau recherche, publiés ou non, émanant des établissements d'enseignement et de recherche français ou étrangers, des laboratoires publics ou privés. 


\title{
The harmonic balance method with arc-length continuation in rotor/stator contact problems
}

\author{
Götz von Groll and David J Ewins \\ Imperial College of Science, Technology $\&$ Medicine \\ Mechanical Engineering Department \\ Exhibition Road, London SW' $2 B X$ \\ United Kingdom
}

\begin{abstract}
There are a variety of abnormal running conditions in rotating machinery which lead to rotor/stator interaction dynamics which, in turn, can cause a rich mixture of effects associated with rub-related phenomena. These effects manifest themselves in the occurrence of multiple solutions for steady-state vibration response scenarios, including amplitude jumps during rotor acceleration, and vibration responses at different/multiple frequencies of excitation forces such as imbalance. This paper describes a numerical algorithm based on the harmonic balance method to calculate the periodic response of a non-linear system under periodic excitation. The algorithm also calculates the stability of the periodic solutions found, marks turning and bifurcation points, and follows a solution branch over varying system parameters via arc-length continuation.
\end{abstract}

\section{INTRODUCTION}

The motivation for this study comes from rotor/stator contact induced vibration problems in turbo-machinery. They can include: rotors touching seals, rotor touching retainer bearings when main active magnetic bearings fail, inter-shaft contact in multiple spool engines, rotor blades contacting the stator, increased bearing clearance through wear or outright bearing failure. In many of these scenarios the rotor continues to rotate and so, depending on the problem, it is often the steady-state response to the out-of-balance excitation forces which is of concern, rather than a particular transient event.

Both the harmonic balance method (HBM) and continuation schemes are wellknown numerical tools to study nonlinear dynamics problems. However, they seem to be used rarely in conjunction with each other in engineering applications, as continuation appears more frequently with time-domain methods, such as shooting or boundary value problem solvers. Recent rotor dynamics examples using timedomain methods with continuation can be found in Sundararajan and Noah $[1,2]$ dealing with squeeze-film-damper and journal bearing analysis and Petrov [3] for shroud/blade friction. Contrary to the shooting method or boundary value prob- 
lems, the HBM is essentially a frequency-domain method. It is not only convenient for purposes of linearisation of systems with small non-linearities [4], but can can also be applied to large non-linearities [5]. Kim et al. [6] analysed the behaviour of rotors with bearing housing clearances using the HBM at discrete speeds but without continuation.

As with most numerical techniques, calculating the Jacobian (most probably by numerical finite difference estimation) is part of solving the equations set up by the harmonic balance method $[7,8]$. It will be shown that the availability of the Jacobian means that the HBM lends itself nicely to studies of the stability of a solution in the frequency domain, i.e. without having to go back into the time domain (Floquet analysis). To the authors' knowledge, such an algorithm for a non-linear system is novel, as only examples of applications to linear time-variant systems have been found in the literature. Naturally, the algorithm described here is not only suitable for rotor/stator contact problems but also applicable to other common non-linear elements in structural dynamics.

\section{HARMONIC BALANCE FORMULATION}

Given the computing resources, the HBM is easily applicable to problems with a large number of degrees-of-freedom (DOFs). Typically, such a problem consists of finite element models of large parts of the structure or substructures where a linear representation is adequate, and some 'problematic' DOFs for special areas, for example where friction, impacts, or other interaction occurs. Usually, the linear DOFs outnumber the nonlinear ones by a large ratio.

The example that will be used later on deals with the dynamics when rotor and stator come into contact. At this stage it does not matter which of the rotor/stator contact scenarios described in the introduction is under investigation. The rotor and stator are modelled as linear structures, and there will be some linear external forces like gravity and out-of-balance. The contact region supplies the nonlinear forcing at a few degrees of freedom on both rotor and stator.

For simplicity, the complete system is split into its linear part, represented by the usual mass, stiffness, damping matrices, with some linear external force vector, $\left\{f_{\mathrm{u}}\right\}$ (for example unbalanced masses), and its nonlinear part, which is represented here as a single force vector $\left\{f_{\mathrm{c}}\right\}$ combining all nonlinear effects (contact between rotor and stator):

$$
[M]\{\ddot{r}\}+[C]\{\dot{r}\}+[K]\{r\}=\left\{f_{\mathrm{u}}(t)\right\}+\left\{f_{\mathrm{c}}(r)\right\}
$$

The harmonic balance method offers an alternative to time-domain methods for analysis of cases where a steady-state, periodic solution to the equation of motion is sought. The general idea is to represent each time history, $r(t)$, by its frequency content, $R(\omega)$, to obtain a set of equations by balancing the terms with the same frequency components and to start an iterative procedure to find the roots of these equations.

An integer variable, $\nu$, is introduced to accommodate possible sub-harmonics of an external excitation frequency, $\Omega$ (for example shaft rotation). The displacements, $r(t)$, and forces, $f(t)$, are represented as truncated Fourier series with $N$ harmonics: 


$$
r(t)=\sum_{n=1}^{N} R_{n} \mathrm{e}^{\mathrm{i} \frac{n \Omega}{\nu} t} \quad f_{\mathrm{c}}(t)=\sum_{n=1}^{N} F_{\mathrm{c}_{n}} \mathrm{e}^{\mathrm{i} \frac{n \Omega}{\nu} t} \quad f_{\mathrm{u}}(t)=\sum_{n=1}^{N} F_{\mathrm{u}_{n}} \mathrm{e}^{\mathrm{i} \frac{n \Omega}{\nu} t}
$$

Substituting these expressions, (2), into the rotor equation of motion, (1), and balancing the harmonic terms yields, for a harmonic $n$ :

$$
\left(-\left(\frac{n \Omega}{\nu}\right)^{2}[M]+\mathrm{i} \frac{n \Omega}{\nu}[C]+[K]\right)\left\{R_{n}\right\}=\left\{F_{\mathrm{c}_{n}}\right\}+\left\{F_{\mathrm{u}_{n}}\right\}
$$

Bringing all $N$ harmonics into one equation can be symbolised as

$$
[\tilde{K}]\{R\}-\left\{F_{\mathrm{c}}\right\}-\left\{F_{\mathrm{u}}\right\}=0
$$

where $[\tilde{K}]$ is a complex block-diagonal matrix of the following form,

$$
-\left(\frac{n \Omega}{\nu}\right)^{2}[M]+\mathrm{i} \frac{n \Omega}{\nu}[C]+[K]=\left[\tilde{K}_{n, n}\right]
$$

and $\{R\}$ and $\{F\}$ are the vectors of Fourier coefficients of displacements and forces, respectively. As the Fourier coefficients, $F_{\mathrm{c}_{n}}$, of the non-linear forces, $f_{\mathrm{c}}$, are functions of the displacements (and thus their respective Fourier coefficients),

$$
F_{\mathrm{c}_{n}}=F_{\mathrm{c}_{n}}\left(R_{0}\left(\omega_{0}\right), R_{1}\left(\omega_{1}\right), \ldots, R_{N}\left(\omega_{N}\right)\right)
$$

equation (4) is non-linear and must be solved iteratively. This iteration process [6] can be sketched as:

$$
R(\omega)^{(k)} \stackrel{\mathrm{FFT}^{-1}}{\longrightarrow} r(t)^{(k)} \rightarrow f_{\mathrm{c}}(t)^{(k+1)} \stackrel{\mathrm{FFT}}{\longrightarrow} F_{\mathrm{c}}(\omega)^{(k+1)} \rightarrow R(\omega)^{(k+1)}
$$

The fast Fourier transform (FFT) and its inverse could be replaced with a (costlier) curve-fitting algorithm that does not rely on an integer number of cycles so that the procedure also works with incommensurate frequency components (quasi-periodic solution). The roots of equation (4) are found using a quasi-newton algorithm, variants of which are widely available in Fortran libraries on the internet or in Matlab's optimisation toolbox.

Finite element models of rotor/stator structures can contain quite a large number of degrees-of-freedom. Setting up equation (4) then leads to a much bigger problem with $2 N+1$ times more unknowns (real and imaginary components for $N$ harmonics and a DC component). Any reduction of the original problem, therefore, leads to a huge saving in computational cost.

\section{REDUCTION}

The harmonic balance method offers an elegant means of reducing the problem order, so that only the non-linear DOFs need to be kept [6]. Clearly being able to keep only the non-linear DOFs vastly increases the speed in cases of linear structures which have a few additional non-linear elements, as is typical for many classes of problems. The equation (3) is re-ordered for every harmonic, $n$ (the subscripts $n$ are omitted in this section for clarity):

$$
\left[\begin{array}{cc}
\tilde{K}_{\mathrm{mm}} & \tilde{K}_{\mathrm{ms}} \\
\tilde{K}_{\mathrm{sm}} & \tilde{K}_{\mathrm{ss}}
\end{array}\right]\left(\begin{array}{c}
R_{\mathrm{m}} \\
R_{\mathrm{s}}
\end{array}\right)=\left(\begin{array}{c}
F_{\mathrm{c}} \\
0
\end{array}\right)+\left(\begin{array}{c}
F_{\mathrm{u}_{\mathrm{m}}} \\
F_{\mathrm{u}_{\mathrm{s}}}
\end{array}\right)
$$


where subscripts $\mathrm{m}$ and $\mathrm{s}_{\mathrm{s}}$ stand for non-linear (master) and linear (slave) degreesof-freedom, respectively. It follows that

and

$$
\begin{aligned}
{[\hat{\tilde{K}}] } & =\left[\tilde{K}_{\mathrm{mm}}\right]-\left[\tilde{K}_{\mathrm{ms}}\right]\left[\tilde{K}_{\mathrm{ss}}\right]^{-1}\left[\tilde{K}_{\mathrm{sm}}\right] \\
\left\{\hat{F}_{\mathrm{u}}\right\} & =\left\{F_{\mathrm{um}_{\mathrm{m}}}\right\}-\left[\tilde{K}_{\mathrm{ms}}\right]\left[\tilde{K}_{\mathrm{ss}}\right]^{-1}\left\{F_{\mathrm{us}_{\mathrm{s}}}\right\}
\end{aligned}
$$

$$
[\hat{\tilde{K}}]\left\{R_{\mathrm{m}}\right\}-\left\{F_{\mathrm{c}}\right\}-\left\{\hat{F}_{\mathrm{u}}\right\}=0
$$

In contrast to the widely-used Guyan reduction, equation (10) is an exact reduction of the original problem as long as the prerequisites for applying the harmonic balance method are met and the number of harmonics included in the decomposition is sufficient. This reduction makes the repetitive task of calculation solutions at various parameters for the continuation of a solution branch much cheaper indeed.

\section{ARC-LENGTH CONTINUATION}

Usually, the system behaviour is of interest over a range of values for at least one parameter (for example speed of shaft rotation), so that the solution has to be calculated at different parameter values consecutively. As time-domain methods seem to be used more often than frequency-domain methods, the use of continuation schemes in conjunction with the harmonic balance method appears to be not as common in engineering applications as, say, with shooting methods. However, arclength continuation is just as applicable in the frequency domain as it is in the timedomain. The task of finding a periodic solution for equation (1) can be transformed into an equivalent root-finding problem, for example by means of finite difference, shooting, HBM (see equation (4))

$$
F(y, \Omega)=0
$$

where $\Omega$ is an independent, externally controlled (exogenous), scalar parameter, such as excitation frequency or rotor speed, over a range in which the solution to equation (1) is of interest.

Using the notation $F_{y}=\frac{\partial F(y, \Omega)}{\partial y}$ and $F_{\Omega}=\frac{\partial F(y, \Omega)}{\partial \Omega}$, the differential of equation (11) can be written as:

$$
\frac{\mathrm{d} y}{\mathrm{~d} \Omega}=-\left(F_{y}\right)^{-1} F_{\Omega}
$$

Choosing $\Omega$ directly as a continuation parameter (called "sequential" or "natural continuation") fails at turning points because of the singularity of $F_{y}$ (see transition from stable to unstable at $\Omega \approx 1.4$ in Figure 2 ). To overcome this limitation, a continuation parameter, $\alpha$, along the arc-length of a solution branch is chosen, so that $y=y(\alpha)$ and $\Omega=\Omega(\alpha)$. From equation (11) one obtains:

$$
F_{y} \frac{\mathrm{d} y}{\mathrm{~d} \alpha}+F_{\Omega} \frac{\mathrm{d} \Omega}{\mathrm{d} \alpha}=\left[\begin{array}{ll}
F_{y} & F_{\Omega}
\end{array}\right]\left(\begin{array}{c}
y^{\prime} \\
\Omega^{\prime}
\end{array}\right)=0
$$

The arc-length, $\alpha$, may be normalised [9], so that the tangent vector, $\left(\begin{array}{l}y^{\prime} \\ \Omega^{\prime}\end{array}\right)$, has unit length:

$$
\left(\frac{\mathrm{d} y_{1}}{\mathrm{~d} \alpha}\right)^{2}+\cdots+\left(\frac{\mathrm{d} y_{N}}{\mathrm{~d} \alpha}\right)^{2}+\left(\frac{\mathrm{d} \Omega}{\mathrm{d} \alpha}\right)^{2}=1
$$


With $\left(y\left(\alpha^{[k]}\right), \Omega\left(\alpha^{[k]}\right)\right)$ denoting a solution previously calculated during continuation, an additional equation can be gained for the additional unknown $\alpha$ by multiplying equation (14) with $(\mathrm{d} \alpha)^{2}$,

$$
\begin{aligned}
0 & =g(y, \Omega, \alpha) \\
& =\left(y_{1}-y_{1}\left(\alpha^{[k]}\right)\right)^{2}+\cdots+\left(y_{N}-y_{N}\left(\alpha^{[k]}\right)\right)^{2} \\
& +\left(\Omega-\Omega\left(\alpha^{[k]}\right)\right)^{2}-\left(\alpha-\alpha^{[k]}\right)^{2}
\end{aligned}
$$

Equation (15) can now be used to parametrise equation (4) by formulating an extended system [10]:

$$
\tilde{F}(y, \Omega, \alpha)=\left(\begin{array}{c}
F(y, \Omega) \\
g(y, \Omega, \alpha)
\end{array}\right)=0
$$

Equation (16) allows the solver to move along the arc-length $\alpha$ of a solution branch. The reader is referred to $[10,11,9]$ for predictor-corrector and step control schemes that facilitate the following of a solution of $\tilde{F}(y, \Omega, \alpha)$ along a range of values for $\alpha$. For simplicity, the results of the previous step $\left(y\left(\alpha^{[k]}\right), \Omega\left(\alpha^{[k]}\right)\right)$ may be used as an initial guess for the next, and a simple step control $\alpha^{[k+1]}=\alpha^{[k]}+\frac{p}{q}$ can be employed, where $p$ is a constant and $q$ is the number of iterations needed for the previous solution. With quasi-Newton solvers an optimal ratio of $\frac{p}{q}$ is suggested by Seydel [10] such that roughly $q=6$ iterations of the quasi-Newton solver are necessary in between arc-length steps. As a first guess initiating the continuation procedure, one may use a random guess or in more diffcult cases find approximate values from a solution obtained previously by time integration. As demonstrated in the numerical example below, there are now no problems in passing turning points and following the overhung part of the solution branch shown in Figure 2. Obtaining information about the stability of such a solution branch being followed is discussed in the following section.

\section{STABILITY}

When HBM with arc-length continuation is employed, there is nothing in the algorithm per se that can warn the user that a particular solution branch followed has stepped over a turning or bifurcation point and the solution has switched stability, from stable to unstable, or vice versa. For example, there might only be a little change in the conditioning of the Jacobian of the system before and after such a change, nor is there a change in the convergence behaviour of the algorithm. This is a practical problem (not a theoretical one, as the Jacobian $F_{y}$ is indeed singular exactly on a turning or bifurcation point) as change in conditioning of the Jacobian could occur far more rapidly than the step-length is able to resolve.

Stability in the time-domain is usually determined by the well-known Floquet multipliers, which are extensively covered in the previously cited text books. It makes sense to employ this method when one operates in the time-domain anyway. For example by using the shooting method, one can determine the stability in parallel to solving for periodic solutions, albeit at the cost of doubling the problem size [1]. However, it is less advantageous when one operates in the frequency domain 
using the harmonic balance method. Many papers employing HBM either ignore the stability issue or revert back to time marching solutions to answer this question. At little computational cost, however, stability can be analysed in the frequency domain with a modification of an algorithm employed for linear time-variant systems. The algorithm is called Hill's method and transforms a linear time-variant system into an eigenvalue problem of a linear time-invariant system [7].

In order to use the same approach for non-linear systems, the stability analysis is carried out by investigating the effect of a perturbation around a periodic solution $r^{\star}(t)$. Although Hill's method for linear systems is documented in text books and the approach to study the stability of nonlinear systems typically involves the linearisation around that solution [10], the following approach of the combination of the two has not been previously encountered in the literature. Let the perturbation be described as $p(t)$, where $p(t)$ consists of a decay term $\mathrm{e}^{\lambda t}$ and a periodic term $s(t)[7,8]:$

$$
p(t)=\mathrm{e}^{\lambda t} s(t) \quad s(t)=\sum_{n=-N}^{N} S_{n} \mathrm{e}^{\mathrm{i} \frac{n \Omega}{\nu} t}
$$

Substituting

$$
r(t)=r^{\star}(t)+p(t)
$$

into equation (1), one obtains

$$
\begin{aligned}
& {[M]\left\{\ddot{r^{\star}}\right\}+[C]\left\{\dot{r^{\star}}\right\}+[K]\left\{r^{\star}\right\}+} \\
&\left(\lambda^{2}[M]\{s\}+\lambda(2[M]\{\dot{s}\}+[C]\{s\})+[M]\{\ddot{s}\}+[C]\{\dot{s}\}+[K]\{s\}\right) \mathrm{e}^{\lambda t} \\
&=\left\{f_{\mathrm{u}}(t)\right\}+\left\{f_{\mathrm{c}}\left(r^{\star}+s \mathrm{e}^{\lambda t}\right)\right\} .
\end{aligned}
$$

By substituting the Fourier representations of $r^{\star}(t), s(t)$ into equation (19), the harmonic components can be balanced in an analogue manner to equation (4):

$$
\begin{aligned}
{[\tilde{K}]\left\{R^{\star}\right\} } & +\left(\lambda^{2}[\tilde{M}]+\lambda[\tilde{C}]+[\tilde{K}]\right)\{S\} \mathrm{e}^{\lambda t} \\
& =\left\{F_{\mathrm{u}}\right\}+\left\{F_{\mathrm{c}}\left(R^{\star}+S \mathrm{e}^{\lambda t}\right)\right\}
\end{aligned}
$$

where $[\tilde{M}],[\tilde{C}]$ are constructed in a similar manner to $[\tilde{K}]$ in equation (5) and $\left\{R^{\star}(\omega)\right\},\{S(\omega)\}$ are the vectors of Fourier coefficients for $r^{\star}(t), s(t)$, respectively.

In what follows, an attempt is being made to find a cost-effective linearisation for the term $\left\{F_{\mathrm{c}}\left(R^{\star}+S \mathrm{e}^{\lambda t}\right)\right\}$ so that equation (20) can be developed further. Consider a variant of equation (4):

$$
\left\{F_{\mathrm{c}}\right\}=[\tilde{K}]\{R\}-\left\{F_{\mathrm{u}}\right\}-\{E(R)\}
$$

where $\{E(R)\}$ is the error in the balancing terms. Developing this as a Taylor series around a known solution of equation $(4),\left\{R^{\star}\right\}$, one obtains

$$
\left\{F_{\mathrm{c}}(R)\right\}=[\tilde{K}]\{R\}-\left\{F_{\mathrm{u}}\right\}-\left[E^{\prime}\left(R^{\star}\right)\right]\left(\{R\}-\left\{R^{\star}\right\}\right)+\text { higher order terms }
$$

with the abbreviation

$$
\left[E^{\prime}\right]=\left[\frac{\partial E}{\partial R}\right]
$$


Substituting equation (22) into (20) and neglecting terms of higher order, equation (20) simplifies to the following eigenvalue problem:

$$
\left(\lambda^{2}[\tilde{M}]+\lambda[\tilde{C}]+\left[E^{\prime}\left(R^{\star}\right)\right]\right)\{S\}=0 .
$$

It is important to note that the term $\left[E^{\prime}\left(R^{\star}\right)\right]$ is already available as a by-product of the quasi-newton solution technique, as it is the Jacobian of the objective function defined by equation (4). In the example below, it was sufficient to approximate the Jacobian numerically by calculating the finite differences in the objective function due to small perturbations (a standard implementation as part of a quasi-newton solver). One is, of course, free to use other methods of determining the Jacobian, ill-conditioned cases might require more sophisticated (robust) methods in order to aid the solver to find the periodic solutions (which is a more general issue of solution convergence than HBM or stability analysis).

Solving for the eigenvalues of equation (23), one obtains a set of $\lambda_{i}$ with real and imaginary parts, where a negative real part indicates stability of the solution, as the perturbation $p(t)$ decays with time, and a positive real part indicates instability. So by solving this eigenvalue problem at the end of the overall iteration procedure, and simply checking if any $\lambda_{i}$ possesses a real part $>0$, one can easily determine whether a periodic solution $r^{\star}(t)$ is unstable. This also helps with finding possible bifurcation points. A change in stability of a solution branch is a sufficient indicator that a turning or bifurcation point has been passed, and the algorithm could be directed to determine the cross-over point within this interval of change more closely. Should this be of interest and the cross-over point found, the rank of $F_{y}$ and $\left[F_{y} F_{\Omega}\right]$ at the cross-over determines whether the point in question is a turning or bifurcation point [10]. If indeed it is a bifurcation point a further solution branch may be followed.

\section{NUMERICAL EXAMPLE}

Although the algorithm described above is easily scalable to systems with a large number of degrees of freedom, a simple modified Jeffcott rotor (Figure 1) is used here for clarity.
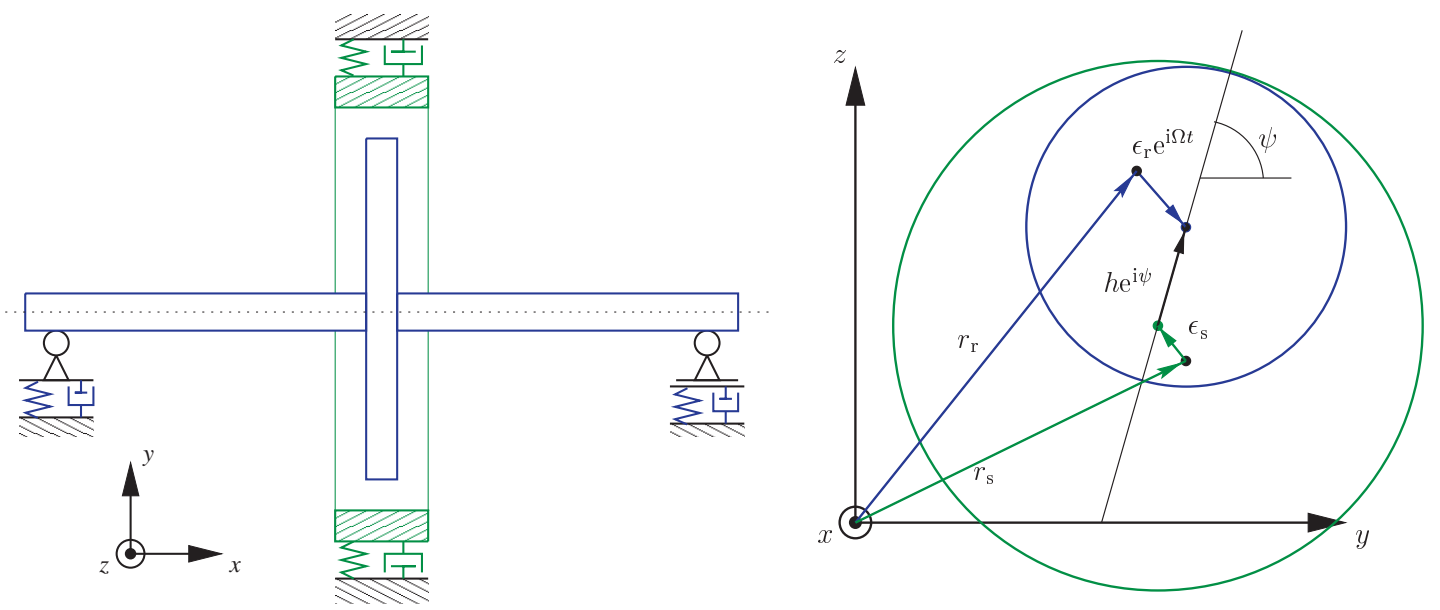

Figure 1. A Jeffcott rotor with stator 
The equations of motion for a Jeffcott rotor interacting with a linear stator structure are:

$$
\begin{aligned}
& m_{\mathrm{r}} \ddot{r}_{\mathrm{r}}+c_{\mathrm{r}} \dot{r}_{\mathrm{r}}+k_{\mathrm{r}} r_{\mathrm{r}}=-f_{\mathrm{c}}+\Omega^{2} m_{\mathrm{r}} \epsilon_{\mathrm{m}} \mathrm{e}^{\mathrm{i} \Omega t} \\
& m_{\mathrm{s}} \ddot{r}_{\mathrm{s}}+c_{\mathrm{s}} \dot{r}_{\mathrm{s}}+k_{\mathrm{s}} r_{\mathrm{s}}=f_{\mathrm{c}}
\end{aligned}
$$

where $r_{\mathrm{r}}, r_{\mathrm{s}}$ are the rotor and stator displacements in the complex plane and $f_{\mathrm{c}}$ is the contact force between rotor and stator,

$$
f_{\mathrm{c}}=k_{\mathrm{c}} \delta
$$

where $\delta$ is the depth of the contact described below in (27) and $k_{\mathrm{c}}$ the local (in this case linear) contact stiffness (for more realistic simulations one would have to choose a nonlinear contact force, see [12]). For the purpose of numerical simulation, a small contact penetration $\delta$ of the rotor and stator rings is allowed. The contact stiffness $k_{\mathrm{c}}$ in this penetration region is being set to a value orders of magnitude higher than the rotor shaft or stator suspension stiffness, so that the penetration depth is orders of magnitude lower than rotor and stator deflections. The contact depth is defined as

$$
\delta(t)= \begin{cases}r_{\mathrm{r}}+\epsilon_{\mathrm{r}} \mathrm{e}^{\mathrm{i} \Omega t}-r_{\mathrm{s}}-\epsilon_{\mathrm{s}}-h \mathrm{e}^{\mathrm{i} \psi} & \text { if }\left|r_{\mathrm{r}}+\epsilon_{\mathrm{r}} \mathrm{e}^{\mathrm{i} \Omega t}-r_{\mathrm{s}}-\epsilon_{\mathrm{s}}\right|>h \\ 0 & \text { otherwise }\end{cases}
$$

where $r_{\mathrm{r}}, r_{\mathrm{s}}$ are rotor and stator displacements, $h$ is the gap size, $\epsilon_{\mathrm{r}}$ a possible offset of the rotor disc and $\epsilon_{\mathrm{s}}$ a stator offset. These entities are depicted in Figure 1.

For the special case of full annular rub with $\epsilon_{\mathrm{r}}, \epsilon_{\mathrm{S}}=0$ and isotropic rotor supports, the equations of motion become quasi-static for pure forward or backward whirl. At a given speed, the steady-state conditions of rotor whirl are such that the radial deflection of the rotor is constant. The only frequency component in the imbalance response spectrum is thus the engine-order speed, $\Omega$, thus $N, \nu=1$. This simple case is used here to illustrate the stability and continuation study, with the following values: $m_{\mathrm{r}}=1, k_{\mathrm{r}}=1, c_{\mathrm{r}}=0.02, m_{\mathrm{s}}=0.1 m_{\mathrm{r}}, k_{\mathrm{s}}=2 k_{\mathrm{r}}, c_{\mathrm{s}}=0.002, \epsilon_{\mathrm{m}}=1$, friction $\mu=0.1$, contact stiffness $k_{\mathrm{c}}=100 k_{\mathrm{r}}(1+\mathrm{i} \mu)$, gap $h=3$. In Figure 2 the magnitudes of the rotor and stator responses $r_{\mathrm{r}}, r_{\mathrm{s}}$ are plotted versus the rotor speed of rotation

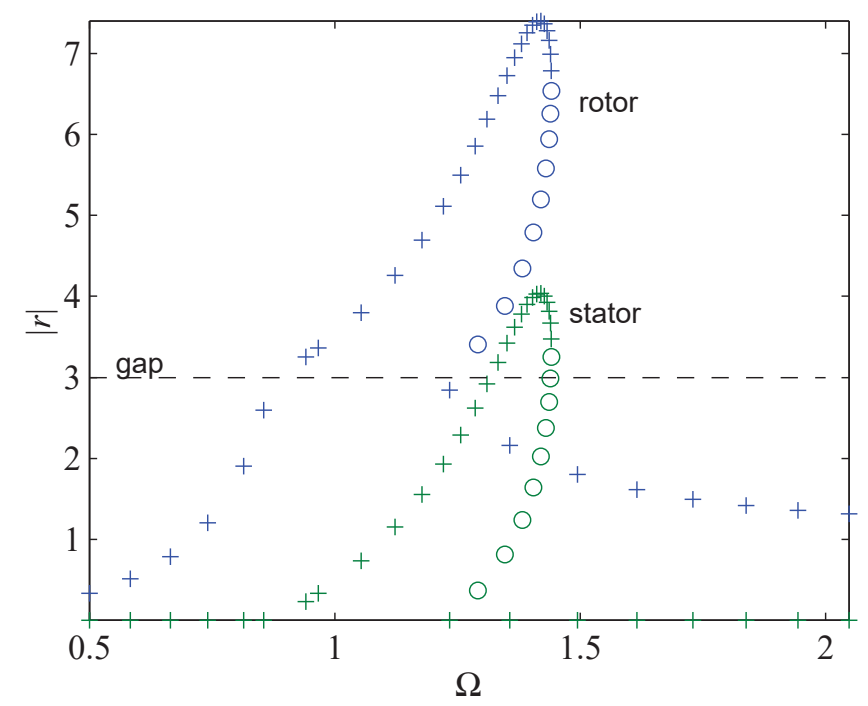

Figure 2. Magnitude of rotor and stator response at constant speed. solution: + stable, o unstable 
$\Omega$. One can see that at speeds $\Omega<0.9$ the rotor imbalance response is too low to overcome the clearance ( $h=3$, dashed line) and rotor and stator are not in contact (stator response zero). As the continuation procedure is started in this speed range with no rotor/stator contact, a simple random guess for the initial conditions is sufficient for solver convergence. At speeds $0.9<\Omega<1.4$ rotor and stator are in contact (non-zero stator response), albeit the overhung part of the curve represents an unstable solution. At speeds $\Omega>1.4$, well past the natural frequency of the rotor, which has been normalised to $\omega_{\mathrm{r}}=1$, the super-critically running rotor loses contact with the stator.

Figure 3 shows a second solution branch at $\Omega>6$, which is not seen in the 1 DOF Duffing type oscillators that display only the overhung behaviour in Figure 2. It must be noted that by following the branch previously discussed, the one that lost contact with the stator and is coming into this picture from the left, there is no indication of the existence of the second branch. The branches of the rotor deflections intersect, but in the whole space of rotor and stator deflections these curves do not come near each other (see stator solution branches), so there is no warning in terms of changes in stability or conditioning of the Jacobian.

This second solution branch was found by brute force, using hundreds of random initial guesses at different speeds, in a quest to find out whether the system could vibrate in an 'inverted' modeshape, where the stator is moving like a hola-hoop around the rotor. The success rate of finding the second branch from the random initial guesses as a whole were poor: many did not converge to any solution, most settled on the solution where rotor and stator were out of contact. The ones that did settle on the second solution branch seemed to settle just as easily in terms of convergence on the unstable part of the branch as on the stable one, albeit a physical system would object to that. Once a solution on that branch is found, it is easy to follow with a continuation scheme.

Making the system more general with $\epsilon_{\mathrm{r}}, \epsilon_{\mathrm{S}} \neq 0$ and non-isotropic rotor and stator mounts gives rise to more complicated motion. Under these circumstance the system

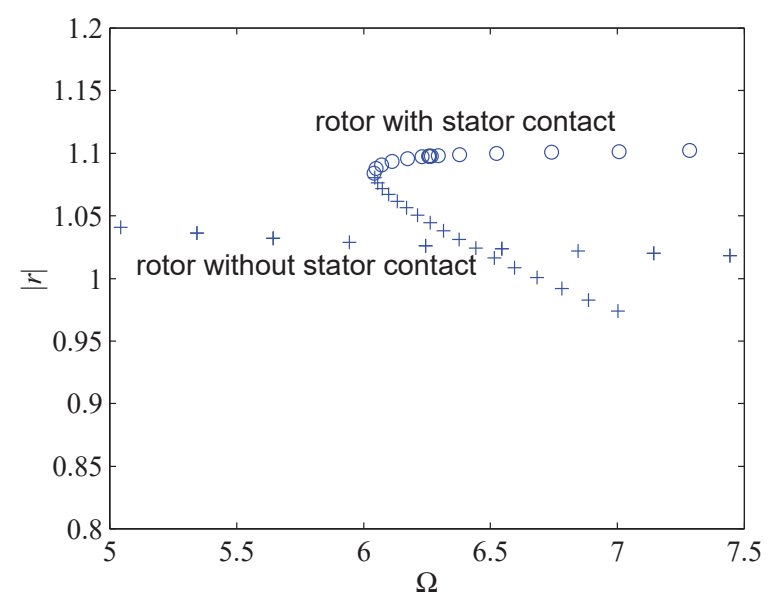

(a) rotor

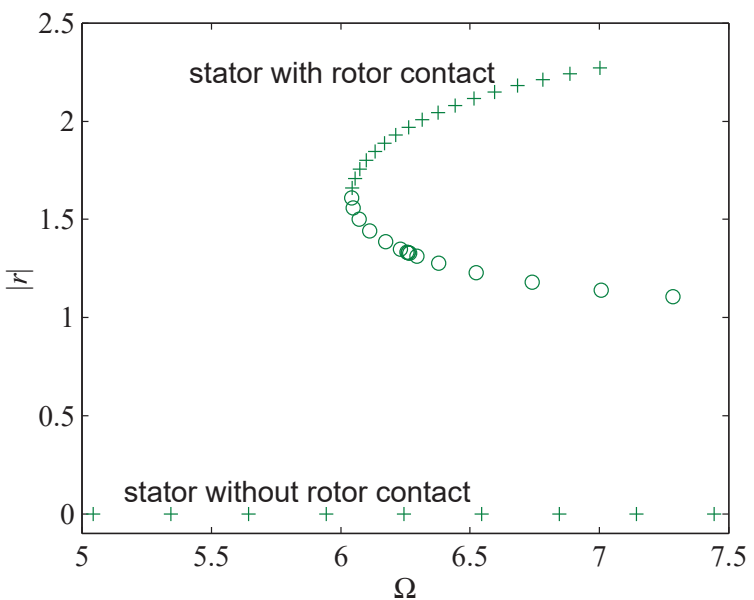

(b) stator

Figure 3. Second solution branch at $\Omega>6$. solution: + stable, o unstable 


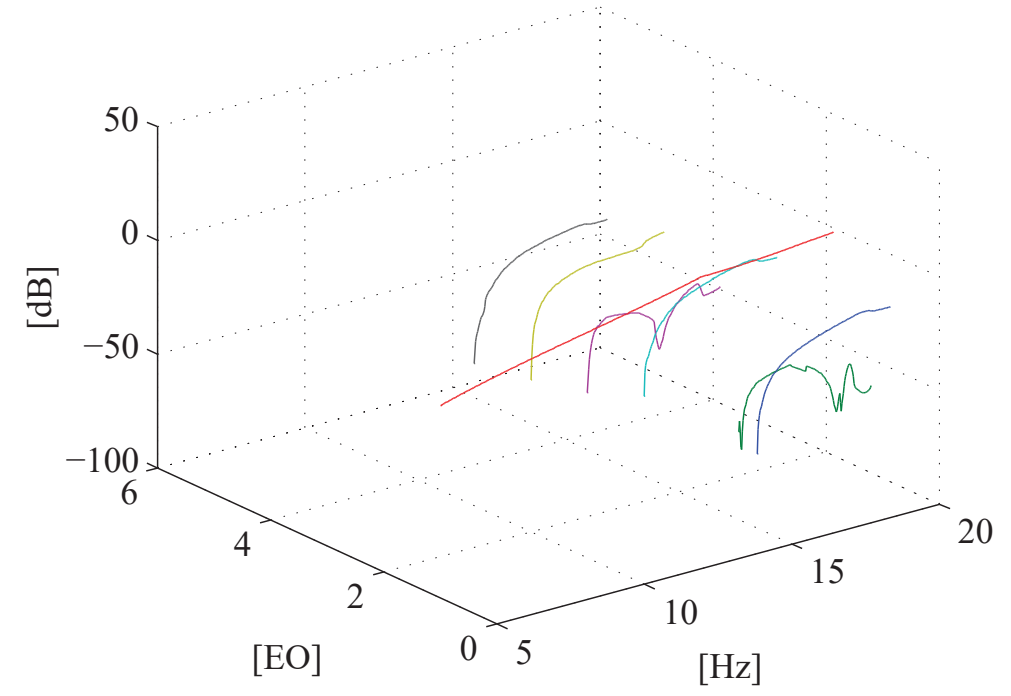

Figure 4. harmonics $1 / 3,1 / 2,1,2,3,4,5$ EO versus speed of rotation $[\mathrm{Hz}]$

has speed regimes where the rotor is rubbing along the stator only over parts of the orbit, causing sub- and super-harmonics to emerge. Even a full annular rub in these circumstances would consist of higher harmonics (but no sub-harmonics, which require intermittent contact). Figure 4 shows the frequency content of the rotor motion at various speeds. The harmonics are expressed in engine order ratios and their magnitude is given in $\mathrm{dB}$. At lower speeds $(<15 \mathrm{~Hz})$ only the $1 \mathrm{EO}(=$ first engine order $=\Omega$ ) component is present, as rotor and stator are out of contact and without the nonlinear forces no other frequency components but $1 \mathrm{EO}$ (due to the imbalance) is expected. As soon as rotor and stator come into contact at higher speeds do the other frequency components start to come into play. $\nu=$ 12 and $n=4,6,12,24,36,48,60$ are chosen to capture the frequency components $1 / 3,1 / 2,1,2,3,4,5 \mathrm{EO}$.

These frequency components are easily confirmed by comparing the results with

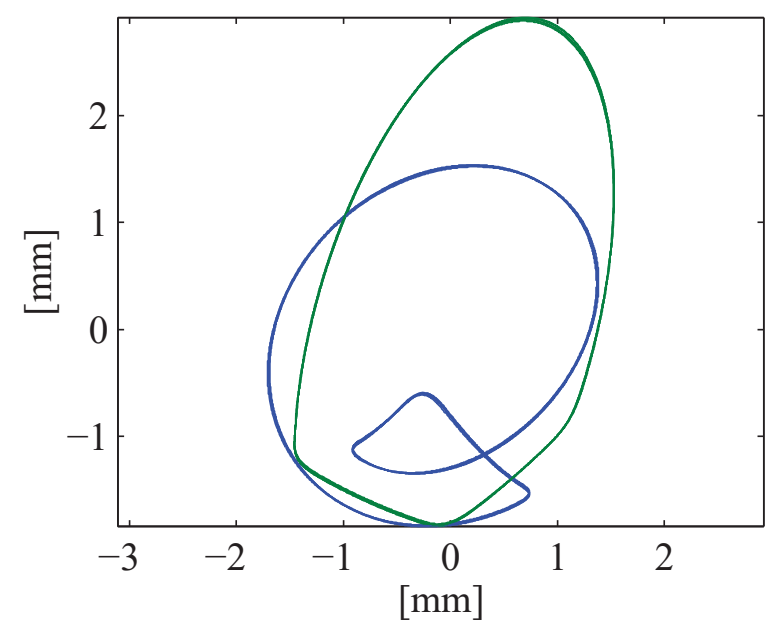

(a) 32 harmonics

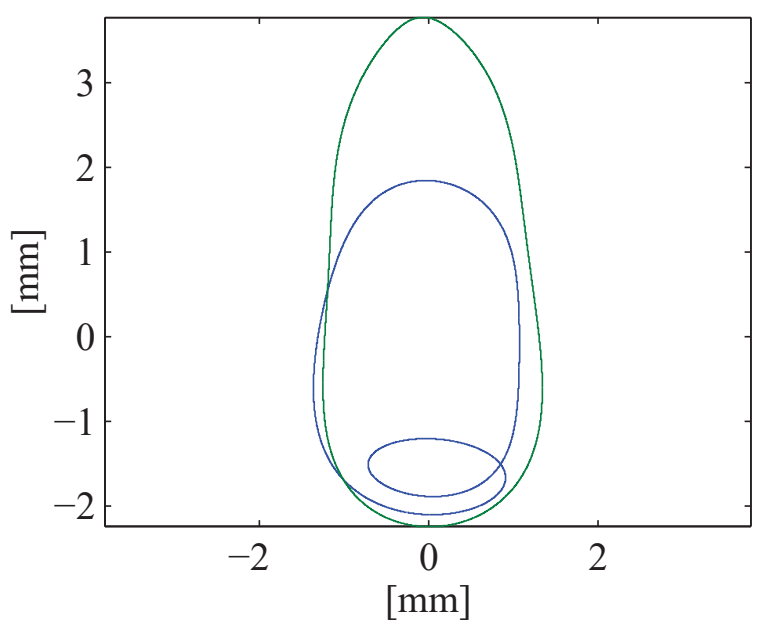

(b) 4 harmonics

Figure 5. HBM rotor and stator results with various numbers of harmonics in the setup 
a time marching solution of the system. Figure 5(a) shows the rotor and stator orbits at a fixed speed $(\Omega=22 \mathrm{~Hz})$ that are obtained in two ways (and plotted on top of each other): time marching and HBM using 32 frequency components $(n=$ $1, \ldots, 32, \nu=2$, the other system parameter values are $m_{\mathrm{r}}=1, k_{\mathrm{r}}=(2 \pi 17)^{2}, c_{\mathrm{r}}=4$, $m_{\mathrm{s}}=2, k_{\mathrm{s}}=(2 \pi 12)^{2}, c_{\mathrm{s}}=3, k_{\mathrm{c}}=100 k_{\mathrm{r}}, h=2, \epsilon_{\mathrm{m}}=0.2, \epsilon_{\mathrm{r}}=0, \epsilon_{\mathrm{s}}=1$.). There is no visible difference between the orbits obtained with time-marching and HBM methods, so for the purpose of this example one may regard the HBM solution with 32 harmonics as exact. However, 32 frequency components is a large number (which are chosen here only to validate that there can be complete agreement between timemarching and HBM results), and it is interesting to see what happens when fewer frequency components are used. Figure 5(b) shows the resulting orbits when the HBM is used with only 4 frequency components in the setup $(n=1, \ldots, 4, \nu=2)$. Not surprisingly, the orbits have a slightly different shape compared to Figure 5(a), but one can see that the fundamental physical effect, namely the sub-harmonic loop, is still retained. When reducing the number of harmonics further to two or only one, the solver only found a physically totally different solution, namely rotor and stator out of contact, where the stator is stationary and the rotor in a simple out-of-balance orbit.

This emphasises the problem to set up the equations to contain those frequencies that the system responds at. It is therefore a method that in general can only be used if some a priory knowledge about a system is available, be it from experience with previous designs, time-marching simulations, or experiments. Measurements from a test rig [13] also show that the physical system in such a rotor/stator contact scenario predominantly responds at a few distinct frequencies, thereby indicating that the HBM with a few selected frequencies is an adequate representation of the physical behaviour.

\section{CONCLUSIONS}

The presented frequency-domain algorithm calculates the periodic solution, traces the solution along a varying parameter and determines the stability of a solution branch. For the investigation of stability previous studies have reverted back to the time-domain. A major motivation for working in the frequency domain is the computational speed advantages it has over time-domain methods.

The solution process of the HBM method itself is less expensive than time-domain methods, and the reduction to the non-linear degrees-of-freedom offers vast savings in large finite element models with only a few non-linear components. It was found that in the given numerical example the harmonic balance method was over 100 times faster than time-domain shooting and boundary-value-problem solving.

HBM also functioned properly in many instances where the time integration routines had difficulties. The source of these difficulties lies in the contact problem. As both rotor and stator have non-negligible mass (and thus dynamics in their own right), the penalty stiffness $k_{\mathrm{c}}$ determines the violence of the rotor/stator impacts and thus tests the robustness and influences the speed of convergence of any chosen method. A more sophisticated contact model can alleviate this problem to a large extent. 
The above mentioned advantages of the algorithm used here make it a worthwhile ingredient in any tool set analysing non-linear systems. However, there are, of course, situations where the harmonic balance method does not find a solution. This is usually an indication that not enough or not the right harmonic components are included in the setup. Furthermore, the fact that a solution has been obtained is not a sufficient condition that no important frequency components have been omitted in the setup. One future enhancement might be to choose a hybrid approach of an initial setup in time-domain, giving an overview of the response spectrum, and continuation in frequency-domain to maximise on the robustness and speed advantages of the HBM.

\section{ACKNOWLEDGEMENTS}

The authors are grateful to Rolls-Royce plc. for providing financial and technical support for this project and for giving permission to publish this work.

\section{REFERENCES}

1. P Sundararajan and S T Noah. 1997 Transactions of the ASME, Journal of Vibration and Acoustics, 119:9-20. Dynamics of forced nonlinear systems using shooting / arclength continuation method - application to rotor systems.

2. P Sundararajan and S T Noah. 1998 Journal of Sound and Vibration, 214(4):695-723. An algorithm for response and stability of large order non-linear systems, application to rotor systems.

3. E Petrov. 1996 in Numerical Methods in Engineering '96, pages 924-930, ECCOMAS Conference, John Wiley \& Sons. Analysis of periodic regimes of forced, essentially nonlinear vibrations of systems of turbine blades.

4. M Géradin and N Kill. 1988 in Fourth International Conference on Vibrations in Rotating Machinery, 627-634. Institution of Mechanical Engineers, United Kingdom. Non-linear dynamic analysis of flexible rotors.

5. Y-S Choi and S T Noah. 1988 Journal of Sound and Vibration, 121(1):117-126. Forced periodic vibraton of unsymmetric piecewise-linear systems.

6. Y-B Kim, S T Noah, and Y-S Choi. 1991 Journal of Sound and Vibration, 144(3):381395. Periodic response of multi disk rotors with bearing clearances.

7. R Gasch and K Knothe. 1989 Strukturdynamik II. Springer.

8. G Genta. 1999 Vibration of Structures and Machines. Springer, third edition.

9. A Nayfeh and B Balachandran. 1995 Applied Nonlinear Dynamics. John Wiley \& Sons.

10. R Seydel. 1994 Practical Bifurcation and Stability Analysis. Springer.

11. T S Parker and L O Chua. 1989 Practical Numerical Algorithms for Chaotic Systems. Springer.

12. M Fumagalli and G Schweitzer. 1996 in Sixth International Conference on Vibrations in Rotating Machinery, 779-788 Institution of Mechanical Engineers, United Kingdom. Measurements on a rotor contacting its housing.

13. G von Groll and D J Ewins. 2000 in Seventh International Conference on Vibrations in Rotating Machinery, Institution of Mechanical Engineers, United Kingdom. On the dynamics of windmilling in aero-engines. 